\title{
ETABLISSEMENT DE LA SPERMATOGENÈSE CHEZ LE CAMPAGNOL DES CHAMPS (MICROTUS ARVALIS) EN FONCTION DE LA DURÉE QUOTIDIENNE D'ÉCLAIREMENT
}

\author{
Lise MARTINET \\ Avec la collaboration technique de Monique Meunier \\ Laboratoire de Physiologie de la Reproduction, \\ Centre national de Recherches zootechniques, Jouy-en-Josas (Seine-et-Oise)
}

Le rôle de la durée d'éclairement sur l'établissement de la spermatogenèse est étudié chez le Campagnol des champs.

Les animaux ont été soumis à quatre photopériodes claires constantes de $5 \mathrm{~h}, 10 \mathrm{~h}, \mathrm{I} 5 \mathrm{~h}$ et $20 \mathrm{~h}$ par $24 \mathrm{~h}$, de la naissance à 70 jours.

Les tests utilisés ont été le poids des testicules et des vésicules séminales, les réserves épididymaires et l'histologie quantitative de l'établissement de la spermatogenèse.

Dès le $20^{\mathrm{e}}$ jour apparaît une différence significative entre les animaux des lots soumis aux photopériodes claires longues ( $5 \mathrm{~h}$ et $20 \mathrm{~h}$ ) d'une part, et ceux soumis aux photopériodes claires courtes ( 5 h et Io h) d'autre part.

Avec tous les critères utilisés, le développement est toujours plus faible pour les courtes durées d'éclairement et il n'existe pas de récupération de la croissance testiculaire avec le temps.

Le seuil de durée de la photopériode claire au-dela de laquelle le développement sexuel est maximum se situe vraisemblablement près de 15 heures.

La durée quotidienne d'éclairement est donc un des facteurs déterminant du cycle saisonnier de reproduction.

\section{INTRODUCTION}

Si dans certains cas, le Canpagnol des champs, Microtus arvalis se reproduit toute l'année (en Allemagne, StErn I952, I953 ; en Russie Maximov, I948), il présente en général un cycle saisonnier de reproduction. 
Pour les uns le facteur principal des variations saisonnières serait le photopériodisme (DELOST, I955) pour les autres ce serait le biotope (STEIN, I952).

De même les résultats des expériences sur le rôle de la lumière sur les fonctions sexuelles du campagnol sont assez contradictoires.

En faisant varier la durée journalière d'éclairement de $I_{5} \mathrm{~h}$ à $9 \mathrm{~h}$, BAKER et RANSon (I932) ont remarqué une diminution considérable de la capacité de reproduction de Microtus agrestis, chez la femelle seulement. Cependant CLARKE (I957) reprenant la même expérience, observe que la réduction d'éclairement de $15 \mathrm{~h}$ à $6 \mathrm{~h}$ pendant des périodes de 30 à 72 jours pertube la spermatogenèse. Au contraire, MARSHALI et WiLIKINSON (I956) n'ont constaté aucune modification de la reproduction chez Microtus orcadensis après une diminution identique de la durée quotidienne d'éclairement, si la nourriture est donnée en abondance.

Devant ces résultats contradictoires, pour connaître, parmi les différents facteurs qui doivent intervenir dans le déterminisme des cycles saisonniers, l'influence exacte de la lumière, nous avons entrepris une étude de l'influence de la durée quotidienne d'éclairement sur l'établissement de la spermatogenèse chez le campagnol lorsque les animaux sont nourris ad libitum.

\section{MATÉRIEL ET MÉTHODES}

A partir de jeunes nés de parents ayant subi auparavant des éclairements journaliers de I 5 heures, quatre lots expérimentaux ont été constitués et soumis respectivement à $5 \mathrm{~h}$, Io h, I $5 \mathrm{~h}$ et $20 \mathrm{~h}$ d'éclairement, de la naissance à 70 jours.

Dans chacun des lots expérimentaux les animaux ont été abattus à l'âge de o, 10, 20, 30, 35, 44 et 70 jours.

A chaque abattage, les animaux, leurs testicules et leurs vésicules séminales étaient pesés. Les testicules étaient fixés au Bouin Hollande et les épididymes broyées suivant la technique de Dounce (1943) pour évaluer les réserves épididymaires.

\section{Etude histologique}

Les testicules ont été coupés à 7 microns et colorés à l'acide périodique Schiff-hématoxyline suivant Clermont (1957). Les diamètres de toutes les sections orthogonales des tubes séminifères présentes sur une coupe ont été mesurés à l'aide d'un micromètre oculaire.

Les numérations des différents types de cellules germinales ont été faites, sur deux ou trois coupes distantes d'au moins $5 \circ$ microns, dans toutes les sections orthogonales des tubes se trouvant à un stade donné du cycle de l'épithélium séminifère. Ce stade est caractérisé par la présence de spermatogonies $A$, de spermatocytes primaires au stade leptotène, de spermatocytes primaires au stade pachytène et de spermatides rondes (stade IX de Clermont, I957, stade I d'OrTavant, I958).

Le nombre réel de cellules germinales est obtenu en corrigeant le nombre de noyaux observés selon la formule d'ABERCROMBIE (I946).

Le nombre total d'une catégorie de cellules germinales présentes dans le testicule a été déterminé à partir d'une formule modifiée de celle d'AMANN ( 1962 ).

Volume testiculaire $\times$ nbre d'une catégorie de cellules germinales par section de tube $\times$ p. 100 sections de tubes au stade étudié

$n=-\quad$ Surface section tubes $\times$ épaisseur section

Nous n'avons pas tenu compte de la rétraction histologique, admettant qu'elle devait être identique pour tous les testicules; nous comptons en fait un nombre proportionnel au nombre exact; ce calcul permet uniquement d'effectuer des comparaisons entre traitements. 


\section{Étude statistique}

Dans les différents lots la comparaison de la croissance pondérale du testicule a été réalisée par des analyses de covariance.

Des analyses de variances comportant une régression ont permis d'étudier les variations du nombre total de cellules germinales en fonction de la durée d'éclairement et en fonction de l'âge.

\section{RÉSULTATS}

La durée de la photopériode claire ne semble pas modifier la croissance générale du campagnol. Par conséquent les variations observées dans les glandes génitales ne sont pas dues à des modifications du poids du corps (tabl. I).

\section{TABLEAU I}

Croissance pondérale des testicules et des vésicules séminales de campagnols soumis à différentes photopériodes

\begin{tabular}{|c|c|c|c|c|c|}
\hline $\begin{array}{c}\text { Age } \\
\text { en jours }\end{array}$ & $\begin{array}{c}\text { Durée } \\
\text { quotidienne } \\
\text { d'éclairement }\end{array}$ & $\begin{array}{c}\text { Nombre } \\
\text { d'animaux }\end{array}$ & $\begin{array}{l}\text { Poids corps } \\
g( \pm \mathrm{sm})\end{array}$ & $\begin{array}{c}\text { Poids testicules } \\
\text { mg ( } \pm \text { : sm) } \\
\text { (moyenne) }\end{array}$ & $\begin{array}{l}\text { Poids vésicules } \\
\text { sêminales } \\
\mathrm{mg}( \pm \mathrm{sm})\end{array}$ \\
\hline $0 \ldots \ldots \ldots \ldots \ldots \ldots$ & - & 9 & $1,8 \stackrel{\therefore}{-} 0,01$ & $1,4+\frac{1}{-L} \quad 0,01$ & - \\
\hline 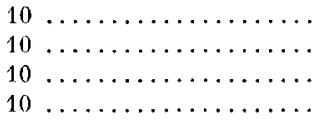 & $\begin{array}{rl}20 & \mathrm{~h} \\
15 \mathrm{~h} \\
10 \mathrm{~h} \\
5 \mathrm{~h}\end{array}$ & $\begin{array}{l}10 \\
10 \\
10 \\
10\end{array}$ & $\begin{array}{l}4,7 \quad \therefore 0,1 \\
3,7 \quad \pm 0,5 \\
3,9 \pm 0,5 \\
5,0 \div 0,2\end{array}$ & $\begin{array}{l}5,0 \doteq 0,3 \\
3,9 \doteq 0,4 \\
5,7 \doteq 0,5 \\
4,8 \pm 0,3\end{array}$ & $\begin{array}{c}- \\
- \\
-\end{array}$ \\
\hline 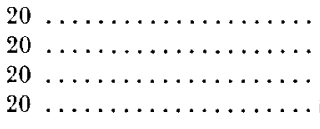 & $\begin{array}{rl}20 & \mathrm{~h} \\
15 \mathrm{~h} \\
10 \mathrm{~h} \\
5 \mathrm{~h}\end{array}$ & $\begin{array}{l}15 \\
16 \\
15 \\
18\end{array}$ & $\begin{array}{l}12,0 \pm 0,7 \\
13,6 \pm 0,7 \\
12,1 \pm 0,6 \\
12,1 \pm 0,5\end{array}$ & $\begin{array}{l}42,9 \text { 上 } 4,7 \\
46,6 \pm 5,9 \\
28,3 \pm \quad 4,4 \\
25,1 \text { ㄴ. } 3,3\end{array}$ & $\begin{array}{l}- \\
-- \\
\cdots \\
-\end{array}$ \\
\hline 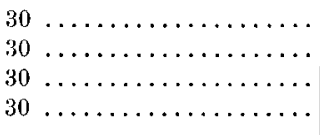 & $\begin{array}{rl}20 & \mathrm{~h} \\
15 \mathrm{~h} \\
10 \mathrm{~h} \\
5 & \mathrm{~h}\end{array}$ & $\begin{array}{l}16 \\
15 \\
15 \\
20\end{array}$ & $\begin{array}{l}18,1 \pm 1,0 \\
18,8 \pm 1,3 \\
18,6 \pm 1,0 \\
17,0 \pm 0,7\end{array}$ & $\begin{array}{r}105,3 \pm 11,0 \\
117,0 \pm 15,2 \\
65,2 \pm 10,0 \\
56,0 \pm 8,2\end{array}$ & $\begin{array}{r}60,9 \pm 15,9 \\
69, \pm \pm 2,7 \\
17,7 \pm 4,7 \\
6,9 \pm 1,6\end{array}$ \\
\hline 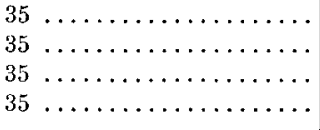 & $\begin{array}{rl}20 & \mathrm{~h} \\
15 \mathrm{~h} \\
10 \mathrm{~h} \\
5 \mathrm{~h}\end{array}$ & $\begin{array}{l}19 \\
15 \\
15 \\
20\end{array}$ & $\begin{array}{l}19,9 \doteq 0,1 \\
19,0 \pm 1,0 \\
20,1 \pm 1,1 \\
16,9 \doteq 0,8\end{array}$ & $\begin{array}{r}135,4 \pm 10,5 \\
110,9 \pm 11,0 \\
75,4 \pm 10,0 \\
45,1 \pm 6,7\end{array}$ & $\begin{array}{r}126,2 \pm 18,3 \\
92,6 \pm 15,3 \\
64,0 \pm 20,1 \\
38,2 \pm 9,4\end{array}$ \\
\hline 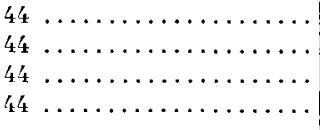 & $\begin{array}{rl}20 & \mathrm{~h} \\
15 \mathrm{~h} \\
10 \mathrm{~h} \\
5 \mathrm{~h}\end{array}$ & $\begin{array}{l}15 \\
15 \\
20 \\
25\end{array}$ & $\begin{array}{l}\geq 1,4 \pm 2,0 \\
25,1 \pm 1,5 \\
20,7 \pm 0,9 \\
23,2 \pm 1,1\end{array}$ & $\begin{array}{r}174,7 \pm 13,2 \\
190,3 \pm 11,6 \\
90,7 \pm 8,6 \\
116,1 \pm 9,6\end{array}$ & $\begin{array}{r}236,0 \pm 3+, 0 \\
237,2 \pm 14,5 \\
83,1 \pm 13,8 \\
115,7 \pm 18,3\end{array}$ \\
\hline 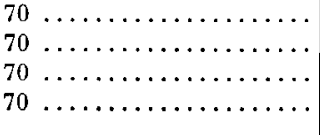 & $\begin{aligned} 20 \mathrm{~h} \\
15 \mathrm{~h} \\
10 \mathrm{~h} \\
5 \mathrm{~h}\end{aligned}$ & $\begin{array}{r}7 \\
10 \\
7 \\
12\end{array}$ & $\begin{array}{l}25,5 \pm 1,1 \\
32,8 \pm 1,0 \\
23,2 \pm 1,6 \\
26,8 \pm 1,7\end{array}$ & $\begin{array}{l}192,6 \pm 12,3 \\
212,7 \pm 10,1 \\
113,3 \pm 20,0 \\
123,9 \pm 7,7\end{array}$ & $\begin{array}{l}320,3 \pm 23,4 \\
362,8 \pm 21,4 \\
182,7 \pm 40,0 \\
187,0 \pm 23,0\end{array}$ \\
\hline
\end{tabular}


A) Résultats concernant la croissance pondérale des testicules de o à 70 jours

Ceux-ci sont consignés dans le tableau I et le graphique I. Si, à Io jours, aucune différence n'existe d'un lot à l'autre, à partir de 20 jours une différence hautement significative $(\mathrm{P}<\mathrm{O}, \mathrm{OI})$ apparaît entre les animaux des 4 lots, la croissance étant plus lente en photopériode courte qu'en photopériode longue.

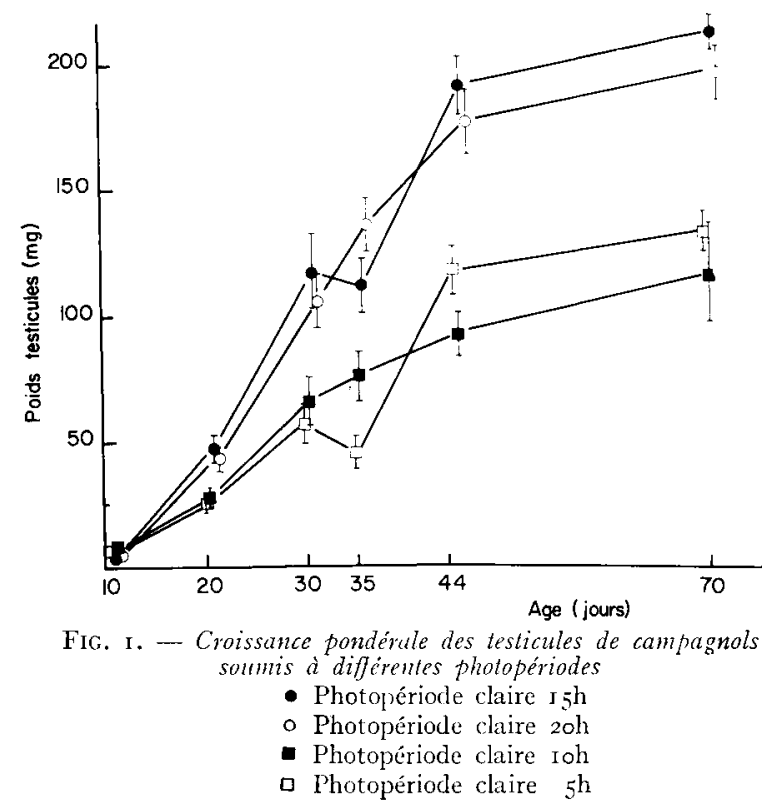

Entre le $30^{\mathrm{e}}$ et le $35^{\mathrm{e}}$ jour, exception faite des animaux recevant 20 heures de lumière par jour, on observe un arrêt ou un ralentissement de la croissance particulière ment net chez les animaux soumis à une photopériode claire de $5 \mathrm{~h}$; au contraire entre 35 et 44 jours se produit une intense récupération de la croissance chez ces mâles.

En outre pour chacun des lots le niveau atteint à 44 jours, quelle que soit la photopériode, ne subit pratiquement plus de variations jusqu'à 70 jours.

Ainsi on obtient deux niveaux de réponse : les animaux soumis à des photopériodes quotidiennes claires de $\mathrm{I} 5 \mathrm{~h}$ à $20 \mathrm{~h}$ ont une croissance testiculaire plus rapide que ceux soumis à des photopériodes claires de $5 \mathrm{~h}$ et $\mathrm{ro} \mathrm{h}$.

\section{B) Croissance des vésicules séminales}

Elle subit les mêmes variations que celle des testicules (tabl. I, graph 2). Chez le campagnol des champs, elle commence au $25^{\mathrm{e}}$ jour environ.

\section{C) Réserves épididymaires}

Les résultats rapportés dans le tableau 2 montrent à 35 jours un retard dans l'apparition des spermatozoïdes dans l'épididyme des animaux soumis à des courtes durées d'éclairement : le pourcentage d'animaux possédant des réserves spermatiques est plus faible pour les photopériodes claires courtes que pour les longues. A 44 et 
70 jours les réserves, chez les animaux des lots à photopériodes claires courtes $(5 \mathrm{~h}$ et ro h), sont très inférieures à celles des animaux des lots à photopériodes claires longues ( $5 \mathrm{~h}$ et $20 \mathrm{~h}$ ).

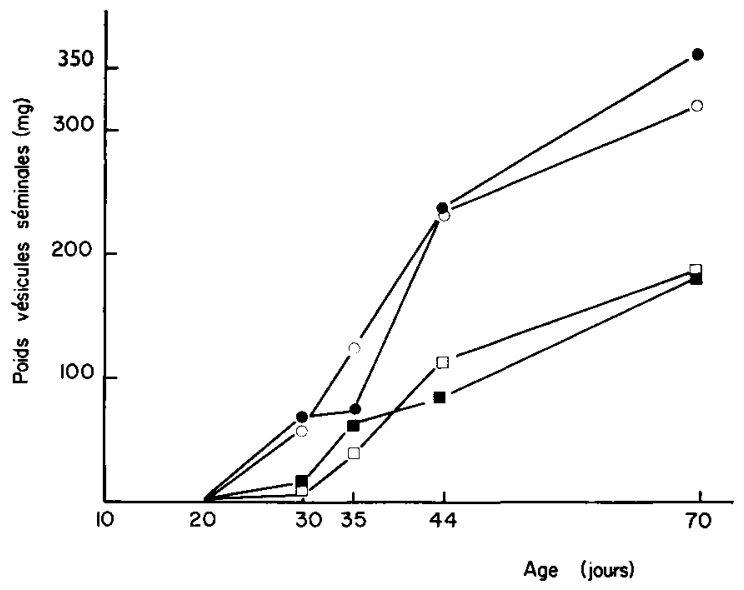

Fir. 2. - Croissance pondérale des vésicules séminales de campagnols sounis à différentes photopériodes

- Photopériode claire $15 \mathrm{~h}$

- Photopériode claire $20 \mathrm{~h}$

- Photopériode claire Io h

a Photopériode claire $5 \mathrm{~h}$

Cependant les spermatozoïdes de ces animaux sont qualitativement fécondants : les mâles ont été testés par accouplement avec des femelles de fertilité connue (tabl. 2) .

\section{TABLEAU 2}

Evolution des réserves épididymaires des campagnols soumis à différentes photopériodes

\begin{tabular}{|c|c|c|c|c|c|}
\hline $\begin{array}{c}\text { Age } \\
\text { en jours }\end{array}$ & $\begin{array}{c}\text { Durće } \\
\text { quotidienne } \\
\text { d'éclairement }\end{array}$ & $\begin{array}{l}\text { Nombre } \\
\text { d'animaux }\end{array}$ & $\begin{array}{c}\text { Animaux possédant } \\
\text { des réserves } \\
\text { épididymaires } \\
\text { en } \%\end{array}$ & $\begin{array}{c}\text { Réserves épidi- } \\
\text { dymaires totales } \\
\text { chez les animaux } \\
\text { possédant } \\
\text { des réserves }\end{array}$ & $\begin{array}{c}\text { Fertilité } \\
\text { (\% gestation après } \\
\text { accouplement des } \\
\text { mâles possédant } \\
\text { des réserves) }\end{array}$ \\
\hline 35 & $\begin{array}{rl}20 & \mathrm{~h} \\
15 \mathrm{~h} \\
10 \mathrm{~h} \\
5 \mathrm{~h}\end{array}$ & $\begin{array}{l}17 \\
15 \\
15 \\
20\end{array}$ & $\begin{array}{l}76 \\
73 \\
60 \\
20\end{array}$ & $\begin{array}{r}3275 \times 10^{3} \\
2925 \times 10^{3} \\
1175 \times 10^{3} \\
400 \times 10^{3}\end{array}$ & $\begin{array}{l}- \\
- \\
-\end{array}$ \\
\hline 44 & $\begin{array}{rl}20 & \mathrm{~h} \\
15 \mathrm{~h} \\
10 \mathrm{~h} \\
5 \mathrm{~h}\end{array}$ & $\begin{array}{l}1 ! k \\
13 \\
16 \\
2 y\end{array}$ & $\begin{array}{r}92 \\
100 \\
88 \\
86\end{array}$ & $\begin{array}{r}16575 \times 10^{3} \\
34125 \times 10^{3} \\
2700 \times 10^{3} \\
10350 \times 10^{3}\end{array}$ & $\begin{array}{l}85 \\
92 \\
87 \\
76\end{array}$ \\
\hline 70 & $\begin{aligned} 20 \mathrm{~h} \\
15 \mathrm{~h} \\
10 \mathrm{~h} \\
5 \mathrm{~h}\end{aligned}$ & $\begin{array}{r}7 \\
10 \\
7 \\
12\end{array}$ & $\begin{array}{l}100 \\
100 \\
100 \\
100\end{array}$ & $\begin{array}{l}52300 \times 10^{3} \\
37687 \times 10^{3} \\
23550 \times 10^{3} \\
15400 \times 10^{3}\end{array}$ & $\begin{array}{r}100 \\
100 \\
87 \\
92\end{array}$ \\
\hline
\end{tabular}

Annales de Biologie animale. - 1963. 


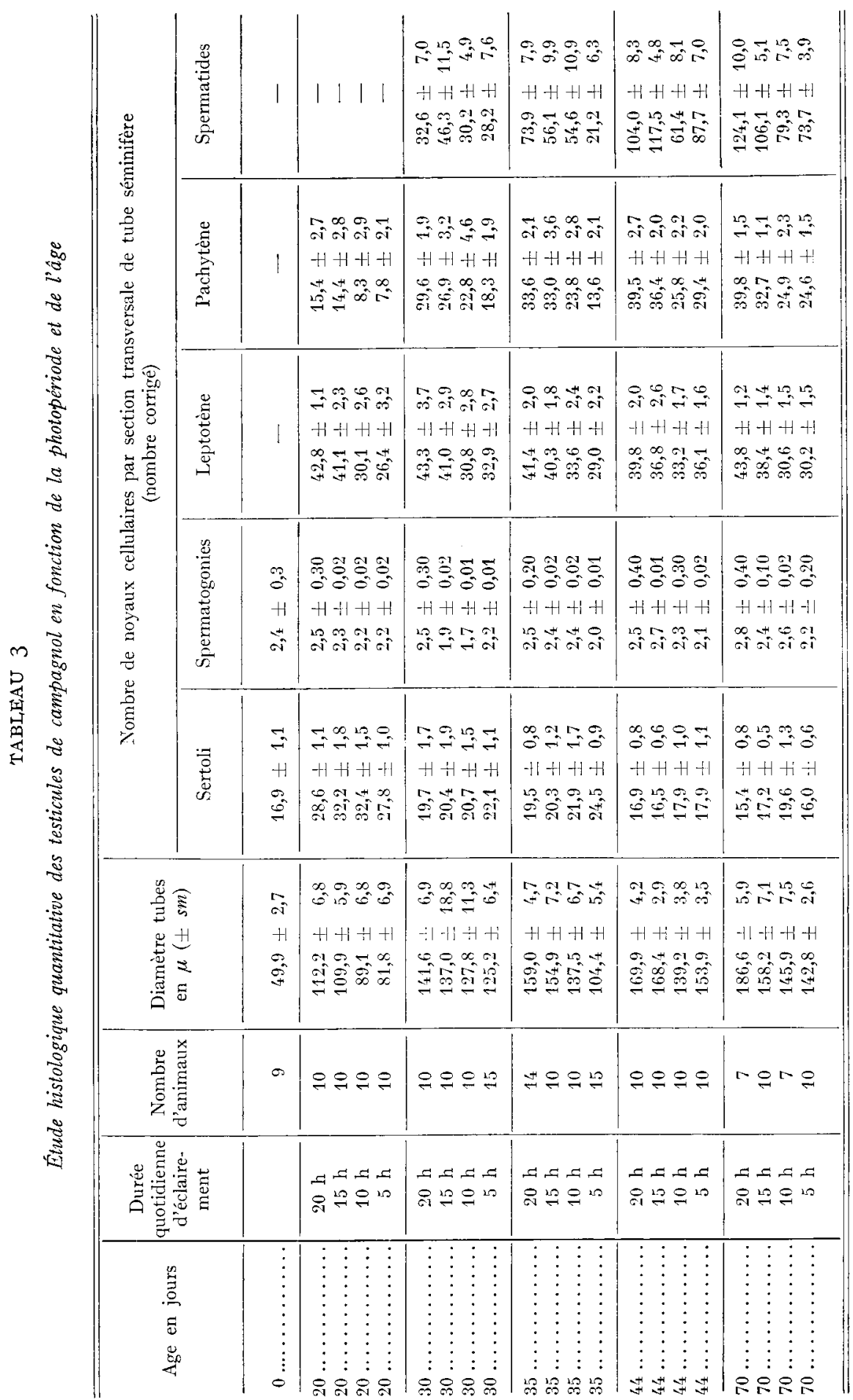


Les résultats concernant les réserves spermatiques sont done comparables à ceux obtenus avec les poids des testicules mais sont décalés dans le temps. L'augmentation importante de ces réserves entre 44 et 70 jours, alors que celle du poids testiculaire est faible, s'explique par la durée des phénomènes spermatogénétiques et du transit épididymaire.

\section{D) Étude histologique de l'épithélium séminifère}

Chez le Campagnol, la chronologie de l'établissement de la spermatogenèse est la suivante : vers 7 jours, les gonocytes migrent contre la membrane du tube séminifère; à Io jours les gonocytes ont disparu, on trouve des spermatogonies $\mathrm{A}$; à I 2 jours apparaissent des spermatocytes au stade leptotène de la prophase méio-

TABLEAU 4

Nombre total arbitraire de cellules germinales au stade leptotène de la prophase méïotique dans le testicule, pour le stade étudié (ce nombre est proportionnel au nombre exact)

\begin{tabular}{|c|c|c|c|}
\hline $\begin{array}{c}\text { Age } \\
\text { en jours }\end{array}$ & $\begin{array}{c}\text { Durée } \\
\text { quotidienne } \\
\text { d'éclairement }\end{array}$ & $\begin{array}{c}\text { Nombre } \\
\text { d'animaux }\end{array}$ & $\begin{array}{l}\text { Nombre total } \\
\text { de leptotìne }\end{array}$ \\
\hline 20 & $\begin{array}{rl}20 & \mathrm{~h} \\
15 \mathrm{~h} \\
10 \mathrm{~h} \\
5 \mathrm{~h}\end{array}$ & $\begin{array}{l}10 \\
10 \\
10 \\
10\end{array}$ & $\begin{array}{l}4380300 \\
4789300 \\
2657700 \\
2102600\end{array}$ \\
\hline 30 & $\begin{array}{rl}20 & h \\
15 & h \\
10 & h \\
5 & h\end{array}$ & $\begin{array}{l}10 \\
10 \\
10 \\
10\end{array}$ & $\begin{array}{l}5971600 \\
6116800 \\
4216700 \\
3678300\end{array}$ \\
\hline 35 & $\begin{array}{rl}20 & \mathrm{~h} \\
15 \mathrm{~h} \\
10 \mathrm{~h} \\
5 \mathrm{~h}\end{array}$ & $\begin{array}{l}10 \\
10 \\
10 \\
10\end{array}$ & $\begin{array}{l}6763000 \\
6003000 \\
3868800 \\
3396900\end{array}$ \\
\hline L't & $\begin{aligned} 20 \mathrm{~h} \\
15 \mathrm{~h} \\
10 \mathrm{~h} \\
5 \mathrm{~h}\end{aligned}$ & $\begin{array}{l}10 \\
10 \\
10 \\
10\end{array}$ & $\begin{array}{l}8153300 \\
8232600 \\
5636300 \\
6337600\end{array}$ \\
\hline 70 & $\begin{aligned} 20 \mathrm{~h} \\
15 \mathrm{~h} \\
10 \mathrm{~h} \\
5 \mathrm{~h}\end{aligned}$ & $\begin{array}{r}7 \\
10 \\
7 \\
10\end{array}$ & $\begin{array}{r}776300 \\
10632500 \\
5382500 \\
6223100\end{array}$ \\
\hline
\end{tabular}

tique ; à 16 jours deux générations de spermatocytes I sont visibles; d'autre part les cellules de soutien ressemblent à des cellules de Sertoli. Vers 20 jours les spermatides apparaissent ; l'élongation des noyaux spermatiques se fait entre 25 et 30 jours. A 35 jours on trouve les premiers spermatozoïdes dans l'épididyme.

Le cycle de l'épithélium séminifère a pu être divisé en huit stades qui correspondent en gros à ceux décrits par ORTAVANT. Le stade pour lequel nous avons fait 
l'étude quantitative des différentes catégories de cellules germinales représente I7,8 p. IOO de l'ensemble des huit stades (MARTINE'T).

L'étude histologique effectuée sur les animaux abattus à $0,20,30,35,44$ et 70 jours a permis d'analyser les résultats obtenus sur les poids testiculaires et les réserves épididymaires (tabl. 3 ).

On constate en effet une diminution du nombre total de chaque catégorie de cellules germinales quand on passe des lots à longue durée d'éclairement ( $5_{5} \mathrm{~h}$ et $20 \mathrm{~h}$ ) aux lots à faible durée d'éclairement.

Il existe en outre au cours de la prophase méiotique une décroissance de la proportion des spermatocytes au stade pachytène par rapport aux spermatocytes a11 stade leptotène quand la durée d'éclairement quotidien est courte ( $5 \mathrm{~h}$ et Io $\mathrm{h}$ )

Nous retrouvons donc l'existence d'une phase critique au stade zygotène de la prophase méotique déjà observée par CLERMONT er MORGENTHALER (I955) chez le rat hypophysectomisé et par OR'TAVAN'T (I956) chez le bélier soumis à de longues photopériodes claires.

Le nombre total de cellules germinales au stade leptotène de la prophase méiotique, présentes dans les tubes au stade étudié, a été calculé (tabl 4).

L'analyse statistique de ces données permet de conclure que la variation de la durée d'éclairement de $5 \mathrm{~h}$ à $20 \mathrm{~h}$ par $24 \mathrm{~h}$ produit un effet significatif : de 20 à 35 jours, la régression est linéaire en fonction de l'augmentation de la période claire. A 44 et 70 jours, il existe une différence très significative entre les lots soumis aux photopériodes claires courtes et aux photopériodes claires longues, mais la réponse n'est plus linéaire.

En outre, le nombre total de cellules germinales varie linéairement en fonction de l'âge de o à 44 jours et pour une même durée d'éclairement. Après 44 jours il n'y a plus d'augmentation du nombre total de cellules germinales quel que soit le niveau atteint sauf pour le lot recevant $I_{5} \mathrm{~h}$ de lumière par 24 heures.

\section{DISCUSSION}

Nos résultats nous permettent de conclure que :

La durée d'éclairement a effectivement une action sur l'établissement de la spermatogenèse chez le Campagnol. En effet, aussi bien les poids testiculaires que les réserves spermatiques et l'étude histologique de l'épithélium séminifère ont montré que les courtes durées d'éclairement ont une action freinatrice sur la spermatogenèse. La durée de la photopériode, qui permet un développement maximum, semble se situer au voisinage de I5 heures ; il n'y a pas d'amélioration au-dessus de ${ }_{5} 5$ heures ni de dégradation supplémentaires en dessous de ro heures, dans les limites de l'expérience.

Cepenđant même avec des durées quotidiennes d'éclairement aussi courtes que 5 heures, l'établissement de la spermatogenèse n'est pas complètement aboli.

Le nombre total de cellules germinales est toujours plus faible chez les animaux des lots soumis à des photopériodes claires de $5 \mathrm{~h}$ et ro h que chez les animaux des lots 5 h et $20 \mathrm{~h}$, au moins jusqu'à 70 jours.

D'autre part, il semble que le nombre de lignées germinales mises en route ne 
soit pas maximum dès le début. En effet on observe une augmentation régulière du nombre total de cellules germinales entre 20 et 44 jours.

Le nombre de lignées germinales serait définitivement influencé par la durée de la photopériode à laquelle l'animal est soumis pendant la phase d'établissement de la spermatogenèse.

Enfin cette expérience nous a permis de constater que l'effet défavorable des photopériodes courtes se manifestait à des stades critiques déjà observés par d'autres auteurs : 1'étude histologique a en effet montré une diminution du nombre des cellules germinales entre les stades leptotène et pachytène de la prophase méiotique ; 1'arrêt de la croissance testiculaire entre 30 et 35 jours pourrait être la conséquence de la difficulté d'établissement de la phase d'allongement des spermatides. Le stade zygotène de la prophase méiotique et l'allongement des spermatides étant des moments critiques chez l'animal hypophysectomisé, l'influence de la durée d'éclairement doit se produire au niveau de l'hypophyse.

Si la durée quotidienne d'éclairement n'est pas le seul facteur en jeu dans le déterminisme du cycle saisonnier chez le campagnol des champs, elle y joue cependant un rôle important pour régler l'établissement de la spermatogenèse.

Cette étude n'a porté que sur la durée quotidienne d'éclairement, elle doit être complétée par le rôle de l'intensité lumineuse sur la spermatogenèse. D'autre part la lumière n'étant pas le seul facteur limitant, il faut envisager son interaction avec d'autres facteurs comme l'alimentation, ou le milieu.

$$
\text { Reçu pour publication en septembre } \mathrm{I}^{6}{ }^{3} 3 .
$$

\section{SUMMARY \\ DEVELOPMENT OF SPERMATOGENESIS IN THE FIELD VOLE (MICROTUS ARVALIS) AS A FUNCTION OF THE DAILY LIGHT DURATION} vole.

The role of light duration in the development of spermatogenesis has been studied on the field

The animals were subjected to 4 illuminations period of 5 hours, Io hours, 15 hours, and 20 hours out of 24 hours, from birth to 70 days.

The parameters used were the weight of the testicles and the seminal vesicles, the epididymous reserves and the quantitative histology of the development of spermatogenesis.

From the zoth day there appeared a significant difference between the animals of the groups subjected to long illumination periods ( 15 and 20 hours) and those subjected to short illumination periods ( 5 and to hours). For all the parameters used, development was always weaker with short light duration and there was no recovery of testicular growth with time. The threshold of the duration of illumination period beyond which sexual development is maximum is probably situated near 15 hours.

The daily light duration is therefore one of the limiting factors which determine the seasonal cycle of reproduction.

\section{RÉFÉRENCES BIBLIOGRAPHIQUES}

Abercrombie M., I 946. Estimation of nuclear population from microtome sections. Anat. Rec., 94,238-248. Amann R. P., I962. Reproductive capacity of dairy bulls. VIII. Direct and indirect measurement of testicular sperm production. J. Dairy Sci., 45, 774-871.

BAKER J. R., RANSON R.M., 1932. Factors affecting the breeding of the field mouse, Microtus agrestis. I light. Proc. Roy. Soc. London, Sec. B., 110, 31 3-32r. 
CLARKE J. R., 1957. Light induced changes in some endocrine organs of the vole (Microtus agrestis). J. Endocrinol., 15, LIV.

Clermont Y., 1957. The stages of the cycle of the seminiferous epithélium of the rat : practical definitions in PAS Schiff hématoxyline sections. Rev. Can. Biol., 16, 45 $1-462$.

Clermont Y., Morgenthaler H., i955. Quantitative study of spermatogenesis in the hypophysectomised rat. Endocrinology, 57, 369-382.

Delost P., I955. Étude de la biologie sexuelle du campagnol des champs (Microtus arvalis Pallas). Arch. Anat. Micr. Morph. Exp., 44, 150-I9o.

Dounce A. L., I943. Further studies on isolated cell nuclei of normal rat liver. J. Biol. Chem., 151, 22I233.

Marshalt A. J., Wilkinson O., 1956. Reproduction in the orkney vole (Microtus orcadensis) under a six hour day length and other conditions. Proc. Zool. Soc. London, 126, 391-395.

Martinet L. Non publié.

Maximov A. A., I948. Fécondité et dynamique du nombre de Microlus arvalis (en russe). Svr Akad Nauk SSSR Ser. Biol., no 1, 73-82.

Ortavant R., I956. Action de la durée d'éclairement sur les processus spermatogénétiques chez le Bélier. C. R. Soc. Biol., 150, 471-474.

Ortavant R., I958. Le cycle spermatogénétique chez le Bélier. Thèse Fac. Sci., Paris.

STEIN G. H. W., I952. Über Massenvermehrung und Massenzusammenbruch bei der Feldmaus. Zool. Jahrb. Abt. Syst., 81, I-26.

STEIN G. H. W., 1953. Über das Zahlenverhältniss der geschlechter bei der Feldmaus, Microtus arvalis. Zool. Jahrb. Abt. Syst., 82, I $37^{-\mathrm{I}} 5^{6 .}$ 